\title{
Implantable Flexible Pressure Measurement System Based on Inductive Coupling
}

\author{
Cristina C. Oliveira*, Student Member, IEEE, Alexandra T. Sepúlveda, Nuno Almeida, Student Member, IEEE, \\ Brian L. Wardle, José Machado da Silva, Member, IEEE, and Luís A. Rocha, Member, IEEE
}

\begin{abstract}
One of the currently available treatments for aortic aneurysms is endovascular aneurysm repair (EVAR). In spite of major advances in the operating techniques, complications still occur and lifelong surveillance is recommended. In order to reduce and even eliminate the commonly used surveillance imaging exams, as well as to reduce follow-up costs, new technological solutions are being pursued. In this paper, we describe the development, including design and performance characterization, of a flexible remote pressure measurement system based on inductive-coupling for post-EVAR monitoring purposes. The telemetry system architecture and operation are described and main performance characteristics discussed. The implantable sensor details are provided and its model is presented. Simulations with the reading circuit and the sensor's model were performed and compared with measurements carried out with air and a phantom as media, in order to characterize the telemetry system and validate the models. The transfer characteristic curve (pressure versus frequency) of the monitoring system was obtained with measurements performed with the sensor inside a controlled pressure vacuum chamber. Additional experimental results which proof the system functionality were obtained within a hydraulic test bench that emulates the aorta. Several innovative aspects, when compared to the state of the art, both in the sensor and in the telemetry system were achieved.
\end{abstract}

Index Terms-Carbon nanotubes, inductive-coupling, pressure sensors, remote monitoring, stent-graft.

\section{INTRODUCTION}

$\mathbf{T}$ HE measurement of aneurysms' intrasac pressure has been reported as a reliable technique to monitor post-endovascular repair (post-EVAR) of abdominal aortic aneurysms (AAA) using prosthetic stent-grafts. The stent-graft

Manuscript received September 6, 2013; revised August 30, 2014; accepted October 6, 2014. Date of publication October 22, 2014; date of current version January 16, 2015. This work was supported by the European Regional Development Fund through the COMPETE Programme (operational programme for competitiveness) and national funds through Fundação para a Ciência e a Tecnologia (Portuguese Foundation for Science and Technology) in the framework of projects MIT-Pt/EDAM-EMD/0007/2008, PTDC/EEI-ELC/1838/2012, and grants SFRH/BD/81476/2011 (C. C. Oliveira) and SFRH/BD/42922/2008 (A. T. Sepúlveda). Asterisk indicates corresponding author.

${ }^{*}$ C. C. Oliveira is with the INESC TEC, Faculdade de Engenharia da Universidade do Porto, 4200-465 Porto, Portugal (e-mail: cristina.oliveira@fe.up.pt).

A. T. Sepúlveda and L. A. Rocha are with the Institute for Polymers and Nanocomposites/I3N, Universidade do Minho, 4800-058 Guimarães, Portugal (e-mail: xanasepulveda@dep.uminho.pt; lrocha@dei.uminho.pt).

N. Almeida was with the Faculdade de Engenharia da Universidade do Porto, 4200-465 Porto, Portugal (e-mail: nunocmalmeida@gmail.com).

B. L. Wardle is with the Department of Aeronautics and Astronautics, Massachusetts Institute of Technology, Cambridge, MA 02139 USA (e-mail: wardle@mit.edu).

J. M. da Silva is with the INESC TEC, Faculdade de Engenharia da Universidade do Porto, 4200-465 Porto, Portugal (e-mail: jms@fe.up.pt).

Color versions of one or more of the figures in this paper are available online at http://ieeexplore.ieee.org.

Digital Object Identifier 10.1109/TBME.2014.2363935 is placed in situ via insertion through the femoral arteries up to the aortic lumen to fit tightly in the bulged or ballooned AAA fraction [1]. This procedure avoids the standard procedure based on open surgery, still mandatory in some cases, but much more invasive than the EVAR approach. Nevertheless, some post-surgery problems, such as endoleaks, graft migration, stent fracture, and enlargement of the aneurysm sac may occur, which require regular monitoring/surveillance and behavioral analysis to avoid the rupture of AAAs and to prevent reinterventions [2]. The pressure in the walls and in the aneurysm sac is one of the most important indicators for post-EVAR patients monitoring. Direct intrasac pressure measurement outperforms imaging techniques in ascertaining either the complete AAA exclusion or the need for future interventions [3].

Remote pressure monitoring systems [4] and results of clinical observation using these systems have been published [5], which show that the use of wirelessly accessed pressure sensors provide useful post-EVAR endovascular leaks observation and help guiding clinical therapy. Until now, two remote pressure sensors have been tested in clinical trials: the EndoSure Sensor (CardioMEMS, Atlanta, GA, USA) and the ImPressure Sensor (Remon Medical Technologies, Caesarea, Israel) [6]. The ImPressure sensor is hand sewed to the outside of a stent-graft and is activated by ultrasound waves from a hand-held probe. In spite of ultrasound being safe and widely used for medical imaging, the measurement requires the use of an ultrasonic gel and direct contact between the skin and the transducer. Another drawback is the high reflection of ultrasound in air-tissue and tissue-bone interfaces, which may lead to difficult communication with the sensor placed in the aneurysm sac. The EndoSure system consists of a simple resonant circuit and a sophisticated external antenna and receiver. The APEX trial demonstrated the efficacy of the EndoSure sensor. This sensor is delivered into the aneurysm sac through its own sheath-adding thus more complexity to the EVAR procedure-and is placed completely separate from the aortic endograft. This way, there is no control on the position of the sensor and it is not possible to have more than one sensor in a patient due to the space it occupies [4].

The existing sensors are randomly placed in the aneurysm sac and only provide information regarding pressure on a single point. We believe that joining sensing capabilities to a stent-graft will benefit the future of EVAR with a more robust technology that enables the placement of more than one sensor, increasing the sensitivity of the measurements and simplifying the deployment procedure.

The alternative being proposed here relies on a flexible capacitive sensor fabricated using aligned carbon nanotubes (ACNTs) embedded in a flexible substrate of polydimethylsiloxane 


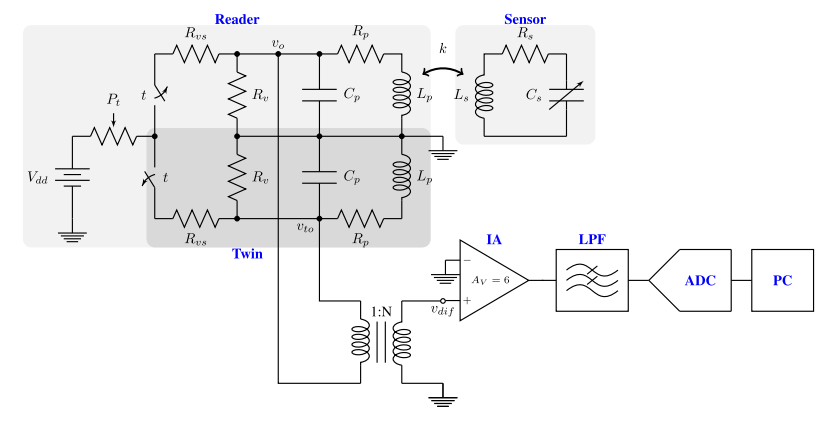

Fig. 1. Schematic of the reader circuit.

(PDMS), a transparent, nontoxic, and biocompatible silicone elastomer. The implantable device (hereafter the pressure sensor) comprises this capacitive sensor and an inductor (antenna) forming together an $L C$ resonant circuit (see Fig. 1). Integrating these pressure sensors within the stent is possible because they can be folded and collapsed together with the stent-graft without sacrificing its structure. Also, given its dimensions, it is possible to actually deploy a cluster of sensors, all already integrated in the stent-graft, each one being identifiable by its own specific resonant frequency. The deployment and monitoring of a cluster of sensors allows obtaining a more accurate observation of the pressure distribution in the aneurysm sac and improves reliability as more than one measurement is obtained simultaneously.

As the working distances between the external reader and pressure sensors are on the order of $\sim 5 \mathrm{~cm}$, frequencies above $50 \mathrm{MHz}$ should be avoided, preference given to those below $20 \mathrm{MHz}$ (15 to $20 \mathrm{~cm}$ penetration depth ensured). This also ensures operation in the antennas near-field [7], where the electromagnetic induction is more efficient. In what concerns protection of the biologic tissues, heat dissipation must be minimized. As tissues' conductivities increase at higher frequencies, lower frequencies are then preferable. However, frequencies below the megahertz range would require larger $L C$ components for the antenna and sensor. In addition, the quality factor of the inductive coupling decreases at low RF frequencies due to skin reflection [8].

This paper addresses the design of the telemetry system, i.e., the wireless pressure sensors and external reading circuit. The electrical model derived for the sensor is presented, and characterization results obtained with simulations performed using the electrical model are compared with those obtained experimentally. The performance of the overall pressure measurement system is evaluated resorting to setups that enable the extraction of the sensors characteristic and emulate the end use of the system. The achieved results are discussed in terms of the system's performance and innovation compared to the state of the art.

\section{Monitoring System Overview}

\section{A. Telemetry System}

The telemetry system comprises an external reader, capable of energizing the passive pressure sensors and detecting the sensor's oscillation frequency, a signal conditioning block having a transformer, an instrumentation amplifier (IA), and a low-pass filter (LPF), as well as a high-speed data acquisition board (ADC) which also provides the interface with a personal computer (see Fig. 1). The sensor receives energy and reflects back its oscillation frequency by means of inductive coupling. Its components can be modeled as passive elements: a variable capacitor $C_{s}$, whose value changes with the applied pressure, connected to an inductor $L_{s}$, implementing a parallel resonant circuit. The reader resorts to a magnetically isolated twin circuit (see in Fig. 1 the circuit included in a dark gray shadowed box) added to the top part of the reader that communicates with the sensor (see in Fig. 1 the circuit included in a light gray shadowed box) in order to provide a differential detection of the reflected oscillation. The sensor's oscillation frequency is obtained after detecting the $v_{o}-v_{t o}$ differential signal using a transformer.

The oscillation frequency [see (1)] is defined by the sensor's capacitance and inductance, as well as the coupling factor $k$ [see (2)], which depends on the reader's coil radius $r_{r}$, the sensor's coil radius $r_{s}$, and the distance between both $x$, as demonstrated in [9]. The $k$ value can be estimated using the data (exact positions and distances of the aorta) from imaging exams that EVAR candidates typically take before the procedure [10]

$$
\begin{aligned}
f_{\mathrm{osc}} & =\frac{1}{2 \pi \sqrt{\left(1-k^{2}\right) L_{s} C_{s}}} \\
k & =\frac{r_{s}^{2} r_{r}^{2}}{\sqrt{r_{s} r_{r}}\left(\sqrt{x^{2}+r_{r}^{2}}\right)^{3}} .
\end{aligned}
$$

The oscillation frequencies are extracted from the frequency spectrum of the captured signal. The frequency spectrum is obtained after a preprocessing step to compensate for noncoherent sampling and the decaying envelope amplitude of the sensor's damped oscillatory response. This step comprises the envelope detection and signal segmentation, followed by smoothing the transient envelope using a Hanning window. The fundamental frequency is extracted from the Fourier transform (by interpolating the frequency domain) [11]. Spectral parameters, such as the signal-to-noise ratio (SNR) and spurious-free dynamic range are used to evaluate the accuracy of the frequency detection, allowing to disregard measurements where the sensor's response is not detected, and providing indication on the quality of the inductive link to the user. Preliminary experimental results obtained with the reader circuit prove its ability to energize an $L C$ network (circuit with the same electrical characteristics of the pressure sensor) and capture the oscillation frequency with good accuracy [12], [13].

\section{B. Pressure Sensors}

The main specifications required for the implantable device (pressure sensor), namely dynamic range, resolution and accuracy, can be retrieved from the maximum values of blood pressure in the human body, required accuracy and maximum errors admitted by the legislation for pressure measurement devices [14]. Taking into account that the cardiac cycle can reach as fast as $180 \mathrm{bpm}(3 \mathrm{~Hz})$, and that the change of rate is below $120 \mathrm{~ms}$, the pressure monitoring system needs to present 


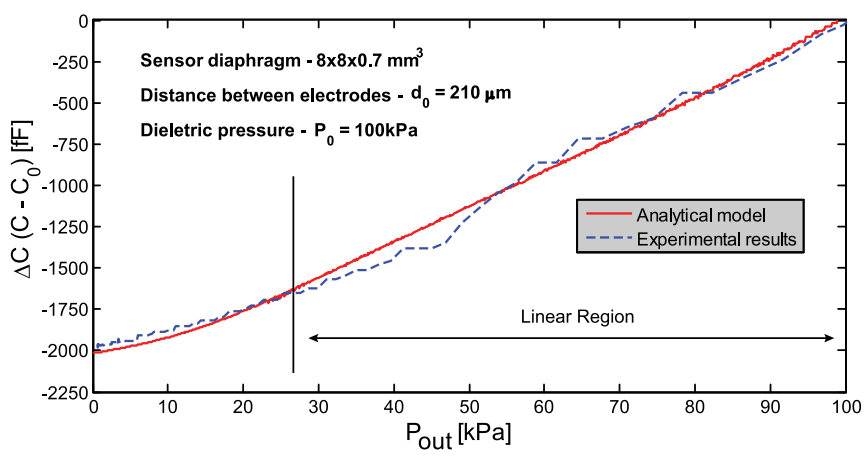

Fig. 2. Pressure sensor transfer characteristic.

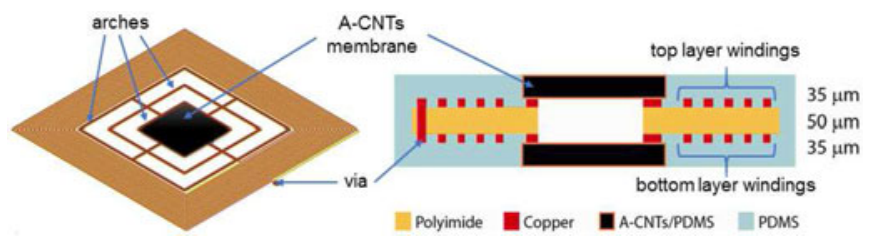

Fig. 3. Overview of the pressure sensor's structure and cross section.

a bandwidth of around $80 \mathrm{~Hz}$. For the aortic aneurysm pressure measurement, the sensor should show a dynamic range between $20 \mathrm{mmHg}(\sim 2.67 \mathrm{kPa})$ and $250 \mathrm{mmHg}(\sim 33.3 \mathrm{kPa})$, referring to the atmospheric pressure, a 1-mmHg resolution and an absolute accuracy below $5 \mathrm{mmHg}$ [15].

The flexible capacitive sensors are fabricated using A-CNTs embedded in a flexible substrate of PDMS, a transparent, nontoxic and biocompatible silicone elastomer, forming a dielectric which is hermetically sealed at ambient pressure [16]-[18].

Fig. 2 shows the measured sensor capacitance versus pressure transfer characteristic. Although the blood pressure in the aneurysm sac ranges from 100 to $130 \mathrm{kPa}$, the pressure sensors were only tested for pressures between 0 and $100 \mathrm{kPa}$ due to technological constraints. The sensor's response fits very well with that predicted by the analytic model [17] and shows relatively good linearity, especially in the region close to the sensor's reference-atmospheric pressure.

Prototypes of the antennas (inductors) attached to the capacitive sensors (see Fig. 3) were fabricated using a DuPont Kapton polyimide film in two different geometries (square and circle). For each geometry, antennas of three sizes were fabricated in order to evaluate different dielectric dimensions (pressure sensor membrane): $4 \mathrm{~mm}, 6 \mathrm{~mm}$, and $8 \mathrm{~mm}$. The antenna is formed with two stacked inductors, one on each side of the polyimide film, interconnected with a via on the outer segment.

The polyimide film has a 50- $\mu \mathrm{m}$ thickness and the copper traces show a $35-\mu \mathrm{m}$ thickness. All antennas' coils are made with $0.1 \mathrm{~mm}$ width traces spaced by $0.1 \mathrm{~mm}$. The three arches between the inductor's coils and the membrane have a $0.25 \mathrm{~mm}$ thickness and are spaced by $1.125 \mathrm{~mm}$. The distance between the inner turn and the largest arch is $0.15 \mathrm{~mm}$ for the square antennas and $0.25 \mathrm{~mm}$ for the circle antennas. Table I displays the outer and inner diameters dimensions ( $d_{\text {out }}$ and $\left.d_{\text {in }}\right)$, the number of turns $\left(N_{\text {turns }}\right)$, as well as the dielectric size (diel-diameter in the case of circles, side in the case of squares).
TABLE I

DIMENSIONS OF THE SENSORS ANTENNAS

\begin{tabular}{lcccccc}
\hline \hline Parameters & C4 & C6 & C8 & S4 & S6 & S8 \\
\hline Geometry & circle & circle & circle & square & square & square \\
$d_{\text {out }}[\mathrm{mm}]$ & 16.3 & 17.5 & 18.8 & 16.3 & 17.5 & 18.5 \\
$d_{\text {in }[\mathrm{mm}]}$ & 10.5 & 12.5 & 14.5 & 10.3 & 12.3 & 14.3 \\
$N_{\text {turns }}$ & 15 & 13 & 11 & 15 & 13 & 11 \\
diel $[\mathrm{mm}]$ & 4 & 6 & 8 & 4 & 6 & 8 \\
\hline
\end{tabular}

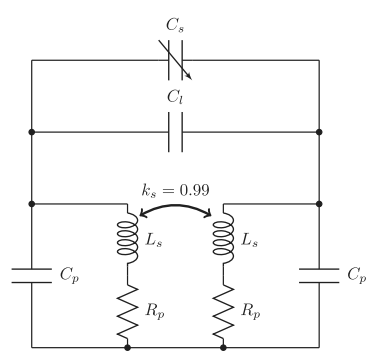

Fig. 4. Electrical model of the pressure sensor.

TABLE II

SENSORS COMPONENTS VALUES

\begin{tabular}{lcccccc}
\hline \hline Parameters & C4 & C6 & C8 & S4 & S6 & S8 \\
\hline$L_{s}[\mu \mathrm{H}]$ & 4.62 & 4.30 & 3.74 & 5.41 & 5.04 & 4.45 \\
$R_{p}[\Omega]$ & 3.58 & 3.37 & 3.09 & 3.99 & 3.87 & 3.58 \\
$C_{p}[\mathrm{pF}]$ & 1.91 & 1.86 & 1.76 & 2.31 & 2.22 & 2.02 \\
$C_{l}[\mathrm{pF}]$ & 19.15 & 18.57 & 17.48 & 33.63 & 32.30 & 29.44 \\
$C_{s}[\mathrm{pF}]$ & 2.23 & 5.01 & 8.90 & 2.83 & 6.38 & 11.33 \\
\hline \hline
\end{tabular}

\section{Pressure Sensor Model}

The sensor's inductor characteristics differ from conventional on-chip inductors or near-field communication RFID antennas, and therefore, a specific electrical model was developed (see Fig. 4). Each layer forms a resonant circuit comprising an inductance $L_{s}$, the parasitic series resistance $R_{p}$, and the parasitic capacitance $C_{p}$ due to the capacitance formed between the traces of the inductor. A parasitic capacitance $C_{l}$, formed between the top and bottom layers traces, is also connected in parallel with the sensor's capacitance $C_{s}$.

The sensors' parameters presented in Table II were analytically calculated using the device's dimensions. The expressions for the calculations of planar spiral inductances presented in [19] were used for the estimation of the inductance of each layer $L_{s}$. The total inductance of the dual-layer planar inductor $L_{\text {total }}$ is almost four times higher than a single-layer inductor with the same dimensions $L_{\text {total }} \cong L_{s(\text { top layer })}+L_{s(\text { bottom layer })}+$ $2 M$. By design, the current flows in the same direction on both layers. This effect plus the proximity and the via interconnecting the inductors assures the mutual inductance $M$ to be almost equal to $L_{s}\left(M=k_{s} \sqrt{L_{s(\text { top layer })} L_{s(\text { bottom layer })}} \cong L_{s}\right)[9]$. The capacitances for the sensor $C_{s}$, the parasitic capacitance between the antenna's layers $C_{l}$, and the parasitic capacitance of each layer $C_{p}$ were estimated using the expression for a parallel plate capacitor. 
TABLE III

OSCILLATION FREQUENCIES FOR \pm 1 PF SENSORS' CAPACITANCE VARIATION

\begin{tabular}{lcccccc}
\hline \hline$f_{\text {osc }}[\mathrm{MHz}]$ & $\mathrm{C} 4$ & $\mathrm{C} 6$ & $\mathrm{C} 8$ & $\mathrm{~S} 4$ & $\mathrm{~S} 6$ & $\mathrm{~S} 8$ \\
\hline$C_{s}-1 \mathrm{pF}$ & 8.04 & 7.95 & 8.07 & 5.68 & 5.71 & 5.93 \\
$C_{s}$ & 7.86 & 7.79 & 7.92 & 5.60 & 5.64 & 5.86 \\
$C_{s}+1 \mathrm{pF}$ & 7.69 & 7.63 & 7.78 & 5.52 & 5.57 & 5.79 \\
$\Delta f$ & 0.352 & 0.323 & 0.286 & 0.154 & 0.139 & 0.139 \\
\hline \hline
\end{tabular}

Simulations using Agilent ADS were performed to estimate the antenna's total inductance, the expected self-resonant frequencies (SRF), and the sensor's oscillation frequency $f_{\text {osc }}$. For antenna characterization purposes, the sensors capacitance $C_{\text {sensor }}$ was discarded from the model, and an S-Parameter simulation was performed to extract SRF and the total inductance of the antennas (at $1 \mathrm{MHz}$ ). For the determination of the sensor's oscillation frequency, the circuit shown in Fig. 1 was simulated (transient analysis) after replacing the simple $R L C$ sensor model by that shown in Fig. 4. The coupling coefficient $k$ established between the reader and the sensor was adjusted in the simulations for all the sensors for a range of distances between 0 and $50 \mathrm{~mm}$ (steps of $5 \mathrm{~mm}$ ), considering the reader antenna to be a spiral square with a 50-mm side length. As a transient simulation is performed, a fast Fourier transform of the signal on node $v_{\text {out }}$ (reader circuit) is computed to extract the sensor's frequency. The results from these simulations will be confronted with experimental measurements in Section V.

To evaluate if the effect of the parasitic layer capacitance masks the change of the sensor's capacitance, a simulation was performed for a variation of the sensors capacitance $C_{s}$ of \pm $1 \mathrm{pF}$, considering a distance between the antennas of $10 \mathrm{~mm}$. Table III displays the variation in frequency $(\Delta f)$ caused by a change of $2 \mathrm{pF}$, which is actually the expected variation of the sensor's membrane capacitance for the working pressure range (see Fig. 2).

\section{EXPERIMENTAL EVALUATION}

This section describes the experiments, as well as the respective setups, performed to characterize the sensors and the complete monitoring system performance. All the results presented here will be discussed in terms of the system's performance and compared with the simulation results in the next section.

\section{A. Sensor Antennas}

Prior to the assembly of the A-CNTs membranes on the flex$\mathrm{PCB}$, the inductance (at $1 \mathrm{MHz}$ ) and the SRF of the antennas were measured with a vector network analyzer. These values will be compared with the values obtained from the S-Parameter simulations in Section V.

\section{B. Experimental Results Obtained Using a Vacuum Chamber}

The performance of the flexible sensors to different pressures and their use with the telemetry system were evaluated using a vacuum chamber. The sensors were subjected to different pressures by applying a pressure varying from 100 to $200 \mathrm{kPa}$. The output of the reader circuit was recorded, while commercial
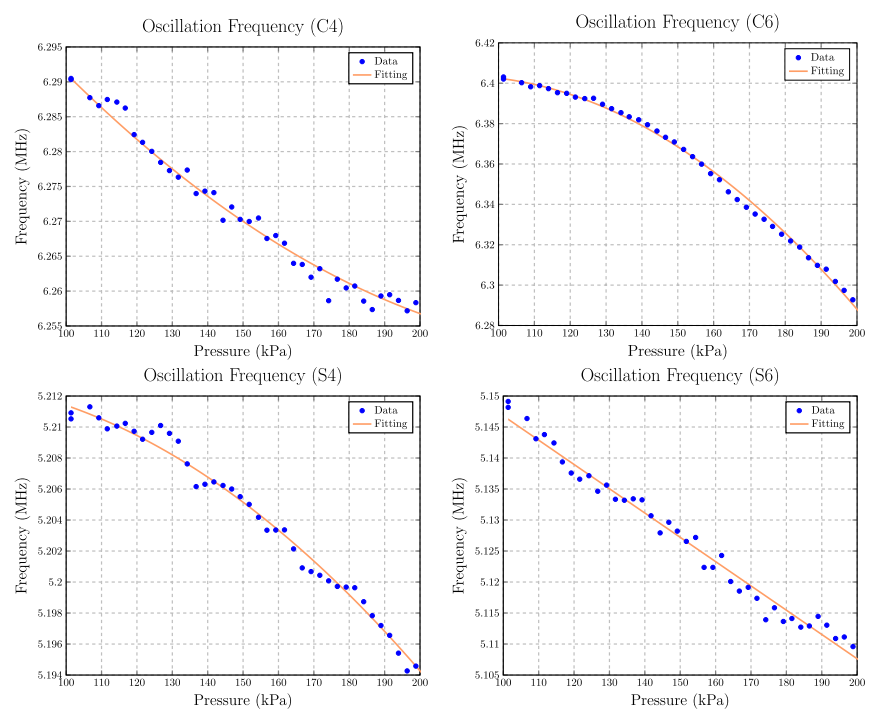

Fig. 5. Sensors' oscillation frequencies measured with the telemetry system after pressure variations performed within a vacuum chamber.

pressure sensors, placed also inside the chamber, were used to record reference values for comparison purposes. Results from the measurements with sensors C4, C6, S4, and S6 are presented in Fig. 5.

\section{Effects of Distance and Biological Tissue in the Frequency Detection}

Since the final application of the system is to read from sensors implanted inside the human body, further experiments were performed in order to assess the behavior of the system in the presence of biological tissue between the antenna and the sensor. A tissue emulating solution (phantom) with electrical (conductivity and permittivity) characteristics similar to the muscle tissue made according to the recipes presented in [20] was used. First, measurements using air as the medium between the reader and the sensor antennas were carried out for different distances ranging from 3 to $25 \mathrm{~mm}$ in steps of $5 \mathrm{~mm}$. The emulating solution was poured in a beaker in order to achieve the desired height, corresponding to the distance between the reader and the sensor. The reader antenna was placed under the beaker and the sensor was inserted in a hermetically sealed bag for protection and laid on top of the phantom solution.

The curves presented in Fig. 6 show the variation of the measured oscillation frequencies of the different sensors, for a range of distances between the sensor and the reading antenna with air and phantom as the dielectric.

\section{Hydraulic Test Bench}

A hydraulic test bench that emulates the aorta was built to test the wireless pressure sensors and the reader system. This model includes the following elements:

1) an electric $2 / 2$-way solenoid valve, connected to a tap water, to simulate the cardiac cycle;

2) a tube to connect the valve to the aneurysm region; 


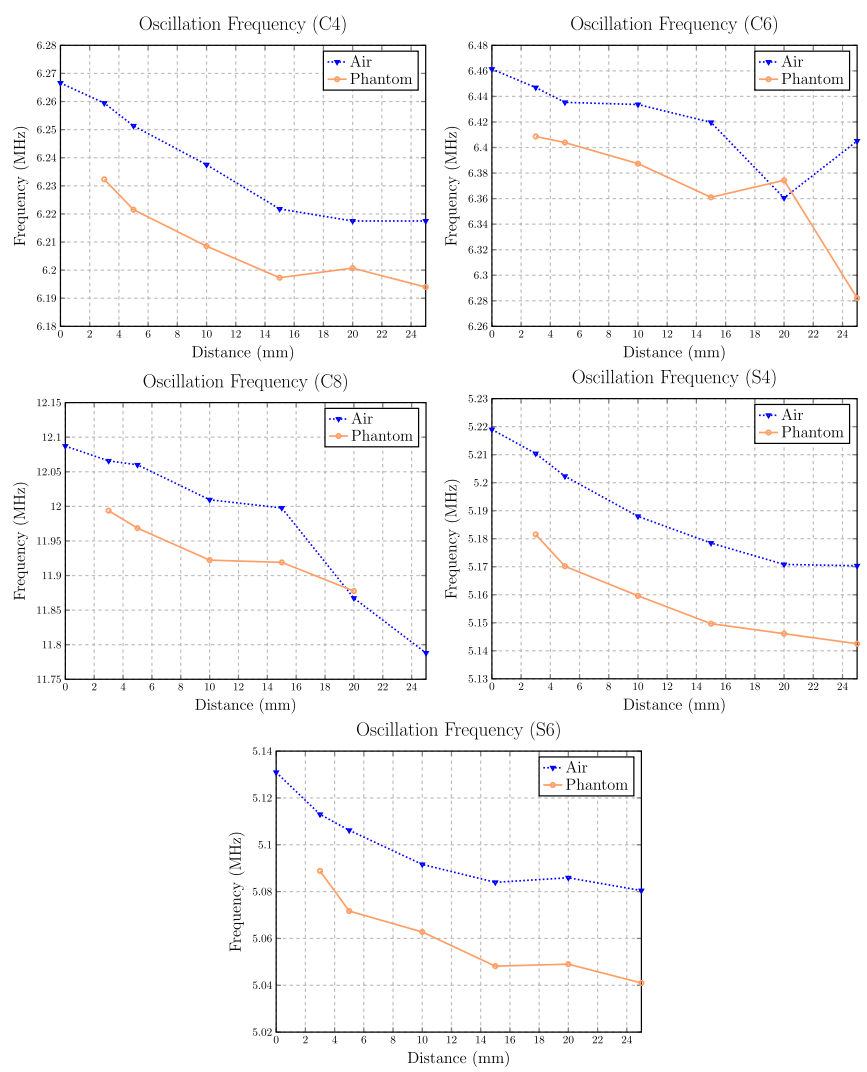

Fig. 6. Measured frequencies of all the sensors with air and phantom as dielectric.

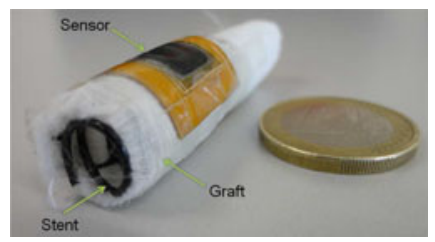

Fig. 7. Stent-graft and the pressure sensor placed on top of the graft.

3) an aneurysm region made with a flexible inflatable material;

4) a stent-graft made with a metal spiral to mimic the stent and a PET material to mimic the graft sewed and placed inside the aneurysm (see Fig. 7);

5) a square $6 \times 6 \mathrm{~mm}(\mathrm{~S} 6)$ wireless pressure sensor placed on top of the stent-graft;

6) a commercial pressure sensor (MPXV5100GC6U from Freescale) placed in the tube right before the aneurysm emulating region (a hole was made in the tube to place the sensor) connected to an acquisition board (NI DAQ USB-6008);

7) the wireless reader system with the reader antenna placed on the aneurysm emulating region.

A diagram of the complete setup is displayed in Fig. 8. The goal of this experiment was to compare the performance of the developed pressure sensor with a commercial pressure sensor. Fig. 9 displays the measured pressure and oscillation frequency

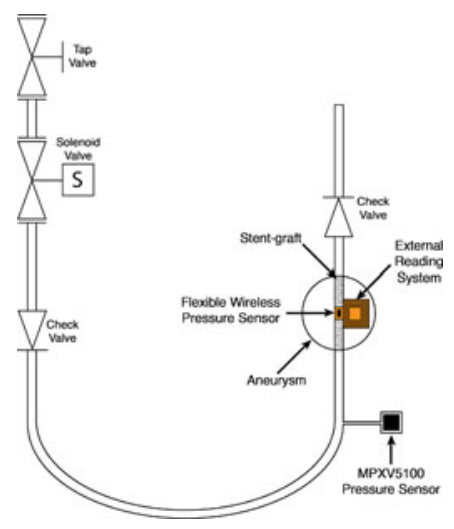

Fig. 8. Diagram of the hydraulic test bench.

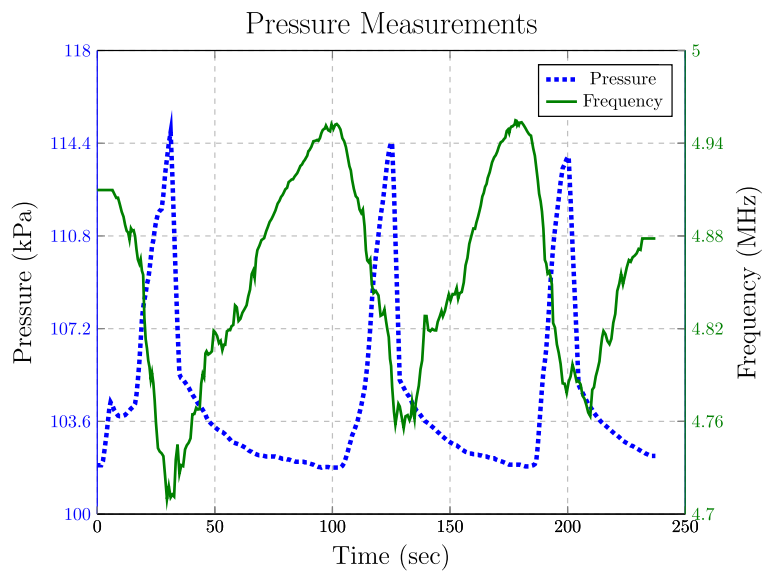

Fig. 9. Pressure measured with a commercial pressure sensor (dotted line) and oscillation frequency from the flexible pressure sensor (solid line), while the water tap was being opened and closed as in the cardiac cycle.

during one trail. In this experiment, the measurements were performed while gradually opening and closing the water tap for six times, to mimic the cardiac cycle.

\section{DISCUSSION}

The next sections discuss the telemetry system performance in terms of the accuracy of the sensor's electrical model, the effect of distance and biological tissue in the frequency detection, the sensor's transfer characteristic curve, and sensors performance in the hydraulic test bench.

\section{A. Accuracy of the Sensor's Electrical Model and the Effect of Distance and Biological Tissue in the Frequency Detection}

Table IV provides a comparison between the simulated and measured parameters of the pressure sensors, such as the antennas' inductance and SRF, and the sensors oscillation frequency after the assembly of the A-CNTs membranes.

The measured inductance value $L_{\text {meas }}$ is lower than the simulated value $L_{\mathrm{sim}}$. However, the antenna is not purely inductive, since the overlap of the top and bottom coils introduces a significant parasitic capacitance, which is responsible for this 
TABLE IV

Comparison Between the Simulated and Measured Parameters of THE PRESSURE SENSORS (INDUCTANCE, SRF, AND OSCILlATION FREQUeNCY)

\begin{tabular}{lcccccc}
\hline \hline Results & C4 & C6 & C8 & S4 & S6 & S8 \\
\hline$L_{\text {sim }[\mu \mathrm{H}]}$ & 18.67 & 17.33 & 15.03 & 22.17 & 20.61 & 18.08 \\
$L_{\text {meas }}[\mu \mathrm{H}]$ & 15.05 & 13.36 & - & 18.08 & 16.12 & 12.78 \\
$\mathrm{SRF}_{\text {sim }}[\mathrm{MHz}]$ & 8.28 & 8.72 & 9.63 & 5.82 & 6.15 & 6.86 \\
$\mathrm{SRF}_{\mathrm{meas}}[\mathrm{MHz}]$ & 7.89 & 7.96 & - & 6.23 & 6.33 & 7.36 \\
$f_{\text {sim }}[\mathrm{MHz}]$ & 7.86 & 7.79 & 7.92 & 5.60 & 5.64 & 5.86 \\
$f_{\text {meas }}[\mathrm{MHz}]$ & 6.32 & 6.43 & 12.01 & 5.19 & 5.09 & - \\
\hline \hline
\end{tabular}

difference, that is, it translates into a measured SRF of the antenna $\left(\mathrm{SRF}_{\text {meas }}\right)$ lower than the simulated value $\left(\mathrm{SRF}_{\text {sim }}\right)$.

Also, the measured oscillation frequency $f_{\text {meas }}$, for a distance of $10 \mathrm{~mm}$ between the sensor and the reading antenna, is lower than that expected from the simulated model $f_{\text {sim }}$, due to the fact that during the assembly of the A-CNTs onto the flex PCB using a special conductive glue, extra parasitic capacitance and resistance are inserted, which cannot be calculated.

The presence of the biological tissue causes a significant shifting of the received signal frequency. This can be due to the change of the medium permittivity to magnetic induction, also affected by the quality factor, as has been reported in [21] and [22]. Regardless of this frequency shifting, when comparing the detected signals in the presence and absence of the phantom, these showed SNRs and amplitude characteristics that allowed an easy detection of the sensor's resonant frequency, in the different cases.

Furthermore, the variation of the detected frequency tends to stabilize after the first $20 \mathrm{~mm}$, meaning that the coupling coefficient $k$ has little influence on the oscillation frequency for distances higher than $20 \mathrm{~mm}$. Since the distance between the abdominal section of the aorta where the stent-graft is placed and the anterior abdominal wall skin is expected to be always higher than $20 \mathrm{~mm}$ [23], the system's performance will not be drastically compromised by the effect of the distance and the biological tissue.

\section{B. Sensors' Transfer Characteristics}

The transfer characteristics of the pressure sensors obtained with the telemetry system have a very defined shape, despite the presence of some small fluctuations in the smaller sensors (see Fig. 5). Equation (3) gives the polynomial relating oscillation frequency with pressure $f_{\text {osc }}(p)$ obtained for sensors $\mathrm{C} 4, \mathrm{C} 6$, and S4 after fitting the measured frequencies with a multiple linear least-squares regression as a function of pressure:

$$
f_{\mathrm{osc}}(p)=c_{1} p^{2}+c_{2} p+c_{3} p .
$$

The data from sensor S6 could be fitted with a linear leastsquares regression due to its more linear behavior [see (4)]:

$$
f_{\mathrm{osc}}(p)=c_{1} p+c_{2} .
$$

Table V displays the regression coefficients.
TABLE V

REGRESSION COEFFICIENTS FOR THE FREQUENCY-PRESSURE CHARACTERISTIC FUNCTIONS OF EACH SENSOR

\begin{tabular}{lcccc}
\hline \hline Coeff. & C4 & C6 & S4 & S6 \\
\hline$c_{1}$ & 1.591 & -9.303 & -0.926 & -391.698 \\
$c_{2}$ & -821.228 & 1646.296 & 106.485 & 5185966.492 \\
$c_{3}$ & 6357389.276 & 6330888.228 & 5210008.700 & - \\
\hline \hline
\end{tabular}

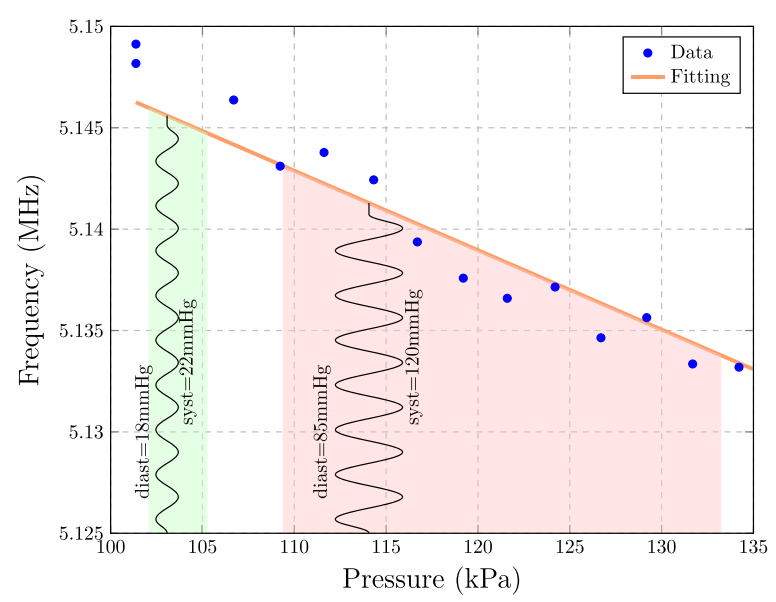

Fig. 10. Transfer function of sensor S6 for the region of interest with the region of pressures in the aneurysm sac expected for a stent-graft without an endoleak (normal) filled in green and for a stent-graft with an endoleak filled in red.

Sensor S6 presents the highest sensitivity in the region of interest, with a frequency variation of approximately $14.981 \mathrm{kHz}$ between 100 and $130 \mathrm{kPa}$. This result was expected since the square sensor has an A-CNT membrane (sensing element) with larger area. The pressure inside the aneurysm sac is expected to be below $40 \mathrm{mmHg}(105.33 \mathrm{kPa})$ in a successfully excluded AAA after the EVAR procedure. If there is an endoleak, the aneurysm sac pressure rises to the levels of the patient arterial blood pressure (ABP), typically between $70 \mathrm{mmHg}(109.33 \mathrm{kPa})$ diastolic and $200 \mathrm{mmHg}$ (126.66 kPa) [3]. Hence, the purpose of our sensors is to distinguish between these two situations. Fig. 10 displays the expected pressure in the aneurysm sac in the normal state (no endoleak), as well as in the presence of endovascular leakage, and compares them with the performance of sensor S6 for the region of interest. A value of $2.28 \mathrm{~Hz}$ on average was obtained for the noise of the sensor plus the complete reading system (resolution of the system) was measured for all the sensors. These data show that for this initial sensor version (not optimized and with large parallel capacitances that greatly reduces sensitivity), it would still be possible to detect endoleaks.

\section{Sensors Performance in a Hydraulic Test Bench}

Although a manual control of the water tap was used and thus there was some uncertainty in the pressure levels, still a variable pressure profile could be obtained that allows us to evaluate the sensor performance. Since the oscillation frequency is inversely 
proportional to the capacitance [see (1)], the highest oscillation frequencies correspond to the lowest pressures (when the water tap is closed). There is a slight delay between the measured pressure and the corresponding frequency shift, which is due to the location of the sensors. Since the water first goes to the commercial sensor and after to the aneurysm sac where the wireless pressure sensor is inserted, the pressure changes are first detected by the commercial sensor, as it is observable in Fig. 9. Also, the detected frequency seems to take longer to reach the values for lower pressures, in opposite to what happens within the commercial sensor that shows faster responses to lower pressures - as the aneurysm emulating sac accumulates water its flux goes from laminar to turbulent.

These proof-of-concept experiments demonstrate the suitability of the small, flexible, and passive sensor along with the readout telemetry system to detect pressure variations.

\section{Telemetry System Calibration}

The telemetry system is being improved in order to include a fault detection system, taking advantage of other physiological signals like the electrocardiogram and ABP, and resorting to measurements of the power and impedance seen from the reader circuit to diagnose deviations in the $L C$ values of the pressure sensor [24], [25]. The reliability of the telemetry system can be further improved by placing a sensor with a fixed $L C$ value, i.e., one whose $C_{s}$ value is not affected by the pressure inside the aortic sac. Taking the measured oscillation frequency for this $L C_{\text {ref }}$ and comparing with (1), the deviation between the measured and expected frequencies can be used to calibrate the remaining sensors, whose responses are affected by the presence of human tissue and tilting. This calibration can be done right after the placing of the stent-graft.

\section{CONCLUSION}

A surveillance mechanism, based on the measurement of the pressure in an aneurysm sac, is an attractive solution to detect possible post-EVAR problems. The pressure measurement system proposed here demonstrates that, after a refinement of the involved technological developments, a simple yet effective and reliable surveillance of post-EVAR is feasible and can be of added value in the treatment of aneurysms. The experimental measurements obtained with the sensors and readout electronics prototypes developed in the lab have shown good agreement with those expected from theoretical computations and simulation results. The tests performed with a hydraulic model validate the proposed telemetry system functionality and shows its feasibility to be integrated into a stent-graft. As pressure sensors are flexible, more than one monitoring sensor can be attached to the stent-graft and the normal procedure to place the stent-graft can be used, as opposed to what happens with the EndoSure solution that requires the insertion of another catheter for the placement of one sensor. Also the EndoSure system resorts to more complex circuitry and algorithms to perform a frequency sweep and extract the pressure measured by the sensor, while the proposed reading circuit is fairly simple and enables the ac- quisition of the pressure values from all the sensors at the same time.

\section{ACKNOWLEDGMENT}

The authors are thankful to Prof. J. G. Mendes from the Departamento de Engenharia Mecânica, Faculdade de Engenharia da Universidade do Porto for assistance with the experiments using the hydraulic model.

\section{REFERENCES}

[1] The United Kingdom EVAR Trial Investigators, "Endovascular versus open repair of abdominal aortic aneurysm," New England J. Med., vol. 362 , no. 20 , pp. 1863-1871, 2010.

[2] B. Katzen and A. MacLean, "Complications of endovascular repair of abdominal aortic aneurysms: A review," Cardiovasc. Interventional Radiol., vol. 29, pp. 935-946, 2006.

[3] B. Sonesson, N. Dias, M. Malina, P. Olofsson, D. Griffin, B. Lindblad, and K. Ivancev, "Intra-aneurysm pressure measurements in successfully excluded abdominal aortic aneurysm after endovascular repair," J. Vascular Surg., vol. 37, no. 4, pp. 733-738, 2003.

[4] F. Springer, R. W. Günther, and T. Schmitz-Rode, "Aneurysm Sac pressure measurement with minimally invasive implantable pressure sensors: An alternative to current surveillance regimes after EVAR?" Cardiovasc. Interventional Radiol., vol. 31, no. 3, pp. 460-467, 2008.

[5] C. J. Parsa, M. A. Daneshmand, B. Lima, K. Balsara, R. L. McCann, and G. C. Hughes, "Utility of remote wireless pressure sensing for endovascular leak detection after endovascular thoracic aneurysm repair," Ann. Thoracic Surg., vol. 89, no. 2, pp. 446-452, 2010.

[6] R. Milner, K. Kasirajan, and E. L. Chaikof, "Future of endograft surveillance," Seminars Vascular Surg., vol. 19, no. 2, pp. 75-82, 2006.

[7] K. Fotopoulou and B. Flynn, "Wireless powering of implanted sensors using RF inductive coupling," Proc. 5th IEEE Conf. Sens., 2006, pp. 765-768.

[8] U. Jow and M. Ghovanloo, "Modeling and optimization of printed spiral coils in air, saline, and muscle tissue environments," IEEE Trans. Biomed. Circuits Syst., vol. 3, no. 5, pp. 339-347, Oct. 2009.

[9] K. Finkenzeller, RFID Handbook: Fundamentals and Applications in Contactless Smart Cards and Identification, 2nd ed. New York, NY, USA: Wiley, Jul. 2003.

[10] J. A. Kaufman, S. C. Geller, D. C. Brewster, C. M. Fan, R. P. Cambria, G. M. LaMuraglia, J. P. Gertler, W. M. Abbott, and A. C. Waltman, "Endovascular repair of abdominal aortic aneurysms current status and future directions," Am. J. Roentgenol., vol. 175, no. 2, pp. 289-302, 2000.

[11] H. Okawara, "SSC applied serial ATA signal generation and analysis by analog tester resources," in Proc. Int. Test Conf., 2009, pp. 1-9.

[12] C. Oliveira, N. Almeida, and J. Machado da Silva, "Inductive coupling system for endovascular aneurysm repair monitoring," Stud. Health Technol. Informat., vol. 177, pp. 101-106, 2012.

[13] C. Oliveira, N. Almeida, and J. Machado da Silva, "A stent-graft endoleakage monitor: Telemetry system based on inductive-coupling transmission for implantable pressure sensors," in Proc. IEEE 3rd Portguese Meeting Bioeng., 2013, pp. 1-4.

[14] "Blood pressure measurement devices (sphygmomanometers)-accuracy," U.S. Dept. Health Human Services, Washington, DC, USA, Rep. CPG 7124.23, Feb. 18, 2005.

[15] J. Potkay, "Long term, implantable blood pressure monitoring systems," Biomed. Microdevices, vol. 10, pp. 379-392, 2008.

[16] A. T. Sepúlveda, F. Fachin, R. Guzmán de Villoria, B. L. Wardle, J. C. Viana, A. J. Pontes, and L. A. Rocha, "Nanocomposite flexible pressure sensor for biomedical applications," Procedia Eng., vol. 25, pp. 140-143, 2011.

[17] A. T. Sepúlveda, R. Guzmán de Villoria, J. C. Viana, A. J. Pontes, B. L. Wardle, and L. A. Rocha, "Flexible pressure sensors: Modeling and experimental characterization," Procedia Eng., vol. 47, pp. 1177-1180, 2012.

[18] A. T. Sepúlveda, R. Guzmán de Villoria, J. C. Viana, A. J. Pontes, B. L. Wardle, and L. A. Rocha, "Full elastic constitutive relation of non-isotropic aligned-CNT/PDMS flexible nanocomposites," Nanoscale, vol. 5, no. 11, pp. 4847-4854, Jun. 2013. 
[19] S. S. Mohan, M. del Mar Hershenson, S. P. Boyd, and T. H. Lee, "Simple accurate expressions for planar spiral inductances," IEEE J. Solid-State Circuits., vol. 34, no. 10, pp. 1419-1424, Oct. 1999.

[20] G. Hartsgrove, A. Kraszewski, and A. Surowiec, "Simulated biological materials for electromagnetic radiation absorption studies," Bioelectromagnetics, vol. 8, no. 1, pp. 29-36, 1987 .

[21] A. Ingelman-Sundberg and E. Odeblad, "Radio frequency absorption in tissues with special reference to diagnostic applications in gynaecology," BJOG: Int. J. Obstetrics Gynaecol., vol. 72, no. 6, pp. 940-945, 1965.

[22] M. Yvanoff and J. Venkataraman, "A feasibility study of tissue characterization using LC sensors," IEEE Trans. Antennas Propag., vol. 57, no. 4, pp. 885-893, Apr. 2009.

[23] M. Narendran and M. S. Baggish, "Mean distance between primary trocar insertion site and major retroperitoneal vessels during routine laparoscopy," J. Gynecologic Surg., vol. 18, no. 4, pp. 121-127, 2002.
[24] C. Oliveira and J. da Silva, "Fault detection system for a stent-graft endoleakage monitor," in Proc. 18th Int. Mixed-Signals, Sens. Syst. Test Workshop, 2012, pp. 17-21.

[25] C. C. Oliveira and J. Machado da Silva, "Towards a dependable cardiovascular surveillance system," in Proc. Int. Conf. Health Informat., Jan. 2014, pp. 118-121.

Authors' photographs and biographies not available at the time of publication. 